\title{
Éducation et croissance
}

Malaisie : un lien fragile

Elsa Lafaye de Micheaux

\section{OpenEdition}

\section{Journals}

Édition électronique

URL : https://journals.openedition.org/ries/1941

DOI : $10.4000 /$ ries. 1941

ISSN : 2261-4265

Éditeur

France Education international

Édition imprimée

Date de publication : 1 avril 2002

Pagination : 157-164

ISBN : 2-84520-553-8

ISSN : $1254-4590$

Référence électronique

Elsa Lafaye de Micheaux, «Éducation et croissance », Revue internationale d'éducation de Sèvres [En ligne], 29 | avril 2002, mis en ligne le 25 novembre 2011, consulté le 05 juillet 2021. URL : http:// journals.openedition.org/ries/1941; DOI : https://doi.org/10.4000/ries.1941

Ce document a été généré automatiquement le 5 juillet 2021.

(c) Tous droits réservés 


\title{
Éducation et croissance
}

\author{
Malaisie : un lien fragile \\ Elsa Lafaye de Micheaux
}

1 L'idée, dominante dans la science économique, qu'il existe une relation forte et solide entre éducation et croissance économique est satisfaisante pour un esprit humaniste : non seulement l'éducation émancipe l'individu mais elle contribuerait au bonheur collectif à travers la croissance économique qu'elle engendre. Dans cet article, nous souhaitons apporter une contribution critique à ce discours en étudiant, dans le cas d'un pays particulier, la Malaisie, choisi pour sa vive croissance économique, le lien qu'entretiennent l'éducation au sens large et cette croissance ${ }^{1}$. Mais comment peut-on démontrer que le rôle de l'éducation dans la croissance malaysienne est fragile, quand les théories récentes de la croissance font intervenir l'éducation comme facteur explicatif déterminant et sachant que les données dont on dispose ne sont ni abondantes, ni fiables?

2 Ce petit pays du Sud-Est asiatique connaît une économie à forte croissance entre 1970 et la crise de 1997. Et dans les années 1990, et on pourrait croire qu'ici, comme ailleurs en Asie, la croissance trouve à s'expliquer par un investissement éducatif particulièrement soutenu et efficace. D'autant plus que l'un des premiers rapports du PNUD met en évidence l'amélioration du développement humain (qui intègre la dimension d'éducation ${ }^{2}$ ) dans ce pays au cours des trente dernières années entre 1960 et $1992^{3}$.

3 Seulement il apparaît qu'il n'y a pas de relation, ou plus précisément pas de relation solide ni claire entre l'effort d'éducation et la croissance malaysienne. Et pour l'établir nous nous fondons sur des recherches successivement menées selon trois directions: l'histoire longue du phénomène, l'analyse macrodynamique (théories des la croissance), et l'approche géographique (on restreint le problème à un lieu particulier, pris comme laboratoire de la croissance nationale). La première et la dernière approches permettent de comprendre ce que la seconde ne fait qu'expliquer à l'aide des lois de la macrodynamique (c'est-à-dire de mécanismes abstraits) de manière très classique en économie. 
4 Nous rendons compte, dans une première section de l'histoire des développements du système productif et du système éducatif malaysien, puis dans la seconde de la difficile application de la macrodynamique et de l'économie géographique au terrain qui est le nôtre, la Malaisie.

\section{Deux mouvements longs au cours de l'histoire}

En étudiant dans l'histoire économique et sociale de la Malaisie les deux mouvements longs du développement de la sphère productive et du système éducatif, nous distinguons deux périodes, l'une (1890-1970) où le lent développement économique, lié à l'exploitation coloniale des ressources naturelles, est inégalitaire et relativement autonome par rapport au développement de l'éducation ; l'autre (1970-1997), phase de croissance très rapide liée à la mise en œuvre de la Nouvelle Politique Économique, où les deux mouvements, pris dans une même logique, sont plus fortement liés.

\section{0-1970 : I'héritage d'une colonie prospère}

6 Depuis les débuts de la colonisation anglaise (fin XIX ${ }^{\mathrm{e}}$ siècle), l'industrialisation et le développement du système éducatif répondent à des logiques spécifiques, du moins jusqu'en 1970. L'étude approfondie de l'histoire nationale, réalisée à partir d'un faisceau de sources et de données disparates mais convergentes, permet d'examiner les logiques à l'œuvre sous la période coloniale et dans les premières années de l'Indépendance. Ces logiques sont tout à fait indépendantes voire contradictoires: l'administration coloniale a pour rôle principal de favoriser la rationalisation et la rentabilité de l'exploitation des ressources naturelles (étain, hévéa) : celle-ci prospère de manière remarquable ${ }^{4}$. En revanche parler de croissance du système éducatif sur le long terme convient difficilement à la situation malaysienne, au moins jusqu'à l'Indépendance (1957), car les divergences de directions prises par les différents acteurs (gouvernement britannique, administrateurs locaux, communautés ethniques) font des créations d'écoles une nébuleuse d'institutions de très petite taille plutôt qu'un système à proprement parler. Bien que le nombre d'élèves scolarisés et le nombre moyen d'années études suivies par les Malaysiens aient augmenté durant cette période, pendant plusieurs décennies les enfants chinois apprirent l'histoire, la géographie chinoises et les principes confucéens, les Malais étudiaient le Coran, et les enfants des écoles tamoules utilisaient les manuels importés d'Inde et de Ceylan. Les élèves des écoles anglaises étudiaient les guerres européennes et les bassins houillers des Midlands d'Angleterre ${ }^{5}$. Il semblerait que les germes de la division sociale aient été semés durant l'époque coloniale, par les choix qui ont été faits d'un système éducatif éclaté ${ }^{6}$.

7 C'est seulement au moment de l'Indépendance qu'un effort de cohérence est entrepris : la structure du système devient alors plus lisible et l'on peut parler alors de développement de l'éducation. Pour autant, la politique éducative n'a pas encore comme objectif la réduction des inégalités et moins encore le souci d'adéquation des formations délivrées aux besoins de l'économie.

8 Pour résumer, la société multiculturelle malaysienne qui se reproduit jusqu'en 1970 est une société encore largement traditionnelle, rurale, et relativement pauvre, dont moins 
d'un tiers travaille dans le secteur moderne mis en place par les Britanniques. La colonisation anglaise laisse de profondes traces institutionnelles et en termes de peuplement géographique, mais n'a pas contribué à l'industrialisation du pays. Enfin, le multiculturalisme (la présence simultanée de grandes communautés religieuses et ethniques distinctes) se traduit par une séparation des systèmes éducatif et politique et par une structuration ethnique des professions et de la géographie humaine. Cette situation éclate de manière paroxystique le 13 mai 1969 lorsque des manifestations politiques dégénèrent en émeutes sanglantes opposant les Malais aux Chinois.

\section{0-1997: les années de forte croissance}

9 À la différence de la période qui précède, une même logique gouverne tous les domaines de l'action politique à partir de 1970. En effet, au lendemain des tragiques événements de 1969, la Nouvelle Politique Économique (NEP) est mise en place : c'est une politique volontariste qui va désormais chercher à industrialiser le pays et à réformer le système éducatif dans le but de réduire les inégalités interethniques et de forger une commune identité nationale. À partir de ce moment, le système éducatif devient un volet de la politique d'affirmative action en faveur des Malais qui comptent la plus grande proportion de ménages ruraux pauvres. Une politique de quotas est mise en place pour favoriser l'accès des Malais à l'enseignement supérieur et à l'emploi : elle est mise en œuvre à la fois dans la fonction publique et dans les entreprises nationalisées ou créées pour l'occasion. Les investissements de l'État poussent alors fortement la croissance.

10 Donc lorsque l'éducation et la croissance économique entrent dans un même projet politique, ce n'est pas pour que l'une de ces dimensions vienne en support de l'autre, mais pour atteindre un but tiers, la construction de l'identité nationale.

11 Le secteur de l'industrie manufacturière se développe véritablement à ce moment. Le résultat en est une forte augmentation du PIB portée par celle des exportations et, au sein du secteur productif, un déclin relatif de l'agriculture au profit de l'industrie. C'est aussi à ce moment que se délocalisent en direction du monde sous-développé les ateliers de production de l'électronique des États-Unis, puis d'Europe et du Japon. Le gouvernement malaysien saisit ici une chance de s'industrialiser sans faire appel à la communauté chinoise (qui est traditionnellement partie prenante du monde des affaires en Malaisie). Le rythme de la croissance malaysienne entre 1970 et 1997 a été de l'ordre de $+8 \%$ par an en moyenne. Trois éléments qualitatifs caractérisent l'industrialisation malaysienne: les exportations sont le moteur de l'industrie, elles viennent de secteurs dominés par les investissements directs étrangers. Enfin le secteur de l'électronique y joue le premier rôle.

En vingt-cinq ans, la Malaisie est ainsi passé de l'état de pays pauvre (pays à bas revenus et à faible développement humain selon les définitions de la Banque Mondiale et du PNUD) à celui de pays situé dans la tranche supérieure des pays à revenu intermédiaire avec 3600 \$ par habitants en $1998^{7}$.

13 Le développement du système éducatif a donc été accaparé par le gouvernement de la NEP pour servir ses buts politiques de changement social, beaucoup plus que dans une perspective de croissance économique. Notamment, le champ de l'éducation est central 
pour construire l'identité nationale à travers l'apprentissage d'une langue commune, la bahasa malaysia.

De 1970 à 1997, les effectifs dans le primaire ont continué de croître lentement par rapport à la période précédente (le taux de scolarisation atteint aujourd'hui près de $100 \%)$. En revanche, l'enseignement secondaire est encore loin d'être généralisé, malgré des progrès continus en termes de nombre d'enfants inscrits, puisqu'en 1995 seulement $57 \%$ de la classe d'âge serait dans le secondaire. L'enseignement supérieur, bien qu'il se soit développé durant la Nouvelle Politique Économique reste cependant fort réduit en 1995, ne concernant in fine que 10,6 \% de la classe d'âge des étudiants, en tenant compte des étudiants à l'étranger qui sont particulièrement nombreux en raison des difficultés pour les non-Malais à intégrer le système universitaire en raison des quotas.

Autres acquis de la période récente : la féminisation de la population scolaire (pour atteindre la moitié des effectifs scolaires dans le primaire et le secondaire en 1995) ${ }^{8}$. Enfin, on constate l'évolution à la baisse du nombre des illettrés dans la population malaysienne. Cette lente diminution est alimentée par la baisse très rapide de leur nombre au sein de la population jeune (la tranche d'âge 15-19 ans), c'est-à-dire grâce au système national d'enseignement 9 .

16 Cependant, certains traits du système de formation malaysien méritent d'être mentionnés comme négatifs. Ces aspects recouvrent essentiellement la question du coût élevé du système dans son ensemble, la tendance à la dégradation de la qualité de l'enseignement et le sentiment de frustration des communautés non-malaises par rapport à l'éducation.

En effet, au-delà de l'effet positif en termes de développement du système et de l'augmentation réelle des chances offertes aux Malais d'avoir un meilleur accès aux études (en particulier aux études supérieures), ces mesures ont eu des conséquences néfastes et inattendues sur la société : les différences en termes de droits ont accentué la conscience que chaque individu a de son groupe ethnique, au lieu de l'affaiblir au profit du sentiment d'appartenance commune à la nation malaysienne. Devenant un sujet de discorde voire de conflit entre groupes ethniques, l'action positive en faveur des Malais visant à réduire les disparités entre groupes ethniques a en réalité contribué à augmenter les divisions. ${ }^{10}$

18 Ainsi, sur le long terme, il apparaît qu'en termes de politique menée, l'éducation et le développement économique sont pris dans des logiques distinctes durant la période coloniale puis visent tous deux à favoriser la cohésion nationale mais ne sont jamais clairement reliés; d'autre part, en termes de réalisation concrète, l'économie et développement du système éducatif, bien que dans une tendance régulière à la hausse, n'ont pas les mêmes dynamiques, ne connaissent pas les mêmes moments de rupture.

\section{Analyse économique de la croissance malaysienne : le laboratoire de Penang}

19 Nous recourons désormais à l'analyse théorique de cette croissance et recherchons dans quelle mesure l'éducation malaysienne que l'on vient de décrire intervient en tant que facteur explicatif. Néanmoins, il faut se souvenir que l'histoire nous a enseigné que l'économie malaysienne n'est pas un tout homogène. Certains secteurs se sont 
développés plutôt que d'autres, et le système éducatif incohérent dans sa mise en place n'est pas un système auquel on a un égal accès selon l'origine sociale et l'appartenance ethnique. D'autre part, géographiquement, le développement économique est inégal et certains lieux concentrent la croissance. Or dans l'analyse macroéconomique ces particularités vont disparaittre, fondues dans les séries statistiques à l'échelle nationale, et l'on va alors chercher à savoir ce que cette mise à distance permet d'avancer qui n'était pas possible en restant au niveau de l'histoire. En revanche, une analyse en termes d'économie géographique permet de préciser les résultats, et comme on le montrera, de les renforcer.

\section{L'analyse macrodynamique}

20 Cette analyse macroéconomique de la croissance se concentre désormais sur la période de forte croissance économique qui a commencé avec la Nouvelle Politique Économique en 1971 et a été stoppée - momentanément du moins - par la crise de l'été 1997.

21 On commence par mesurer ainsi la contribution de l'éducation à la croissance selon les méthodes néoclassiques : la principale source de croissance de 1971 à 1987 peut être attribuée à la croissance du capital et du travail, le premier étant de loin le facteur le plus important ${ }^{11}$. Entre 1971 et 1987 , le capital a été le véritable moteur de la croissance malaysienne. Au regard de ces chiffres, la croissance de l'efficacité globale des facteurs (donnée par la productivité totale des facteurs) a été dérisoire. Ainsi, la croissance rapide de cette période a-t-elle été alimentée par la croissance en volume des facteurs, en particulier du capital et non par la croissance de leur productivité. Des conclusions identiques sont à formuler pour les années 1986-1991 concernant le secteur industriel. Ce résultat rejoint les dernières analyses du développement asiatique auxquelles Krugman a donné un large écho: il n'y a pas de miracle asiatique, la croissance est due à l'augmentation du volume des facteurs et non de leur efficacité productive.

22 Ensuite, nous confrontons le modèle de croissance endogène de Lucas, 1988 à la réalité malaysienne. En effet, c'est là le modèle dominant sur le sujet «éducation et croissance » dans les années quatre-vingt dix, il est impossible de ne pas s'y arrêter longuement. Or on démontre que le modèle de Lucas est réfuté par le cas malaysien: l'éducation n'est pas le moteur de la croissance économique.

23 Enfin, le modèle évolutionniste de Nelson et Phelps (1966) nous a amenée à nous interroger sur la relation entre éducation et croissance non plus à travers l'augmentation de la productivité du travail mais à travers l'innovation. Ces auteurs font l'hypothèse très suggestive que le niveau d'éducation (et non plus son taux de croissance) engendre la croissance économique par la capacité à acquérir des idées nouvelles qu'il donne aux travailleurs. Ce modèle est renforcé et enrichi par le modèle de [Romer 1990] ${ }^{12}$.

24 Cependant, il n'est pas possible de tester empiriquement cette hypothèse au niveau de la Malaisie faute d'indicateurs utilisables dans ce pays mais on peut observer néanmoins que le niveau d'éducation de la population active n'est pas assez haut pour qu'il soit possible d'intégrer et de maîtriser les technologies importées par les investissements directs étrangers. 


\section{Approche géographique : l'État de Penang}

Pour chercher encore plus avant comment intervient l'éducation dans la croissance, nous sommes passée à l'étude du problème en proposant de lui attribuer une dimension géographique. Notre troisième partie s'attache à l'étudier au niveau d'un territoire, l'État de Penang. Penang représente en réalité un laboratoire de la croissance nationale dont il possède tous les traits caractéristiques : l'industrie de l'électronique, industrie exportatrice et portée par les investissements directs étrangers, s'y est concentrée depuis les années 1970.

On cherche alors à expliquer le fonctionnement du cluster ${ }^{13}$ de l'électronique de Penang en s'interrogeant sur la place de la formation (l'éducation de base et la formation continue) dans le phénomène. Nous avons scindé en deux ce problème: on s'est demandé d'abord pourquoi les filiales des multinationales de ce secteur s'y sont-elles établies, et ensuite pourquoi un réseau de sous-traitants locaux en relation avec ces filiales est apparu à Penang alors que les liens entre les entreprises locales et les firmes étrangères sont si réduits au niveau de l'ensemble du pays.

L'explication du premier phénomène ne fait pas intervenir le niveau d'éducation de la population locale dans les raisons de l'implantation. Ensuite, l'analyse en termes de district industriel, basée sur un travail de terrain sur le site de Penang, permet de répondre au deuxième aspect du problème: le succès économique et la capacité d'innovation spécifique de Penang repose sur la présence et l'activité d'un centre de formation continue (résultant de la coopération des multinationales), et sur la richesse des relations interpersonnelles au sein du district. Cependant, il s'agit pour l'essentiel d'une activité reposant sur une main-d'œuvre peu qualifiée et la relative faiblesse du niveau de formation initiale (en particulier technique) limite les possibilités de développement industriel et technologique du site, qui reste de ce fait bloqué au stade de l'assemblage et du test de semi-conducteurs.

Quel que soit l'angle sous lequel on entreprend d'aborder le problème, il apparait que dans la vive croissance de ces vingt dernières années en Malaisie, ce n'est pas l'éducation qui a tenu le rôle clé.

29 Si l'on se replace dans le long terme, on peut montrer que le développement du système éducatif échappait déjà largement à la rationalité économique qui poussait l'administration britannique à exploiter les ressources naturelles de la colonie. La seule cohérence que partagent au cours du siècle le système éducatif et le système productif est celle de la division ethnique. La politique qui se met en place alors, la New Economic Policy, vise à promouvoir à toute force l'accès de la population malaise à ce secteur économique moderne dont elle est écartée. À partir de 1970 le pays s'industrialise, poursuit et améliore le développement de son système éducatif. La croissance est rapide, ce qui permet à tous les groupes sociaux de connaître une hausse du niveau de vie sur plus de vingt-cinq ans, jusqu'à la rupture de la crise de l'été 1997. Mais si les ambiguïtés de la Nouvelle Politique Économique n'ont pas empêché l'économie malaysienne de croître rapidement, elles ne l'ont pas non plus poussée.

Ainsi, au sortir d'une recherche sur le lien entre éducation et croissance qui s'est apparentée à une traque tant la relation a semblé parfois faire défaut, nous devons admettre qu'en Malaisie ce lien est fragile, ténu. 


\section{BIBLIOGRAPHIE}

ABDELMALKI L., COURLET C. (dir). (1996) : Les nouvelles logiques du développement. Globalisation versus localisation. Paris, L'Harmattan, $415 \mathrm{p}$.

AGHION P., HOWITT P. (1998) : Endogenous Growth Theory. London, Edwards Elgar Publishers, $450 \mathrm{p}$.

ANDAYA B., ANDAYA L. (1982) : A History of Malaysia. Londres, MacMillan. 350 p.

ANDERSON B. (1996) : L'imaginaire national. Paris, La Découverte. 213 p.

BOYER A. (1992). L'explication en Histoire. Lille, Presses Universitaires de Lille. 284 p.

BOYER R. (1989) : «Économie et histoire : vers de nouvelles alliances ? " Annales ESC, $n^{\circ} 6$ nov-déc, pp. 1397-1426.

BRAUDEL F. (1950) : «Pour une économie historique ». Revue Économique, vol. 85, n 1. pp. 37-44.

CARRY A. (1993) : «Eléments d'analyse de la conjoncture éducative en long terme ». Journées AFSE, Dijon. 26 p.

CROUCH H. (1996) : Government and Society in Malaysia. Cornell U Press, London. 266 p.

DAVID P. (1994) : « Why are Institutions the 'Carriers of History' ? Path Dependance and the Evolution of Conventions, Organizations and Institutions » Structural Change and Economic Dynamics, vol. 5, n 2. pp. 205-220.

DENISON E.F (1962) : The Sources of Economic Growth. The Brooking Institution, Washington.

DIEBOLT C. (1997) : «Education and Economic Growth in France and Germany in the 19th and 20th Centuries. » The Fourth Oxford International Conference on Education and Development. $17 \mathrm{p}$.

JOMO K.S. (1987) : A Question of Class : Capitalism, the State and Uneven Development in Malaysia. Singapour, Oxford University Press. 353 p.

JOMO K.S., FELKER G., RASIAH R. eds., (1999) : Industrial Technology Development in Malaysia : Industry and Firm Studies. Routledge. 400 p.

KIM J.I., LAU L.J. (1994) : « The Sources of Economic Growth of the East Asian Newly Industrialized Countries ». Journal of Japanese and International Economies, 8. pp. 235-271.

KRUGMAN P. (1994) : « The Myth of Asia's Miracle », Foreign Affairs 74, traduction française in La mondialisation n'est pas coupable., 1998. Paris, La Découverte. pp. 163-182.

LOH Ph. (1972) : Seed of Separatism : educational policy in Malaya 1874-1940. Oxford U. Press, Kuala Lumpur.

LUCAS R.E. (1988) : « On the Mechanics of Economic Development ». Journal of Monetary Economics 22. pp. 3-42.

MINGAT A., TAN J.P. (1992) : Education in Asia. A Comparative Study of Cost and Financing. World Bank, Regional and Sectoral Studies. 204 p.

NELSON R.R., PHELPS E.S. (1966) : «Investment in humans, technological diffusion and economic growth ». American Economic Review, $\mathrm{n}^{\circ}$ 61. pp. 69-82.

SOLOW R.M. (1956). « A contribution to the theory of economic growth ». Quaterly Journal of Economics, vol. 70, February. pp. 65-94. 
SOLOW R.M. (1957) : « Technical Change and the Agregate Production Function ». The Review of Economics and Statistics, vol. 39, $\mathrm{n}^{\circ}$ 3. pp. 312-320.

THAM S.Y. (1995) : « Productivity, Growth and Development in Malaysia ». The Singapore Economic Review, vol. 40, n 1. pp. 41-63.

YOUNG A. (1995) : « The Tyranny of Numbers ». Quaterly Journal of Economics, (110) August. pp. 641-680. vol. 38. pp. 964-973.

\section{NOTES}

1. L'objet du présent article est de rendre compte de la démarche et des principaux résultats de notre thèse, Éducation et croissance en Malaisie, étude d'un lien fragile. Université de Dijon, décembre 2000.

2. L'éducation, mesurée par le taux d'alphabétisation des adultes et le nombre d'années de scolarité moyen, constitue l'un des quatre arguments de l'indicateur composite du développement humain (IDH).

3. PNUD, Rapport sur le développement humain, 1994 : l'IDH malaysien a augmenté en valeur absolue de 0,463 entre 1960 et 1992. Il est passé en trente ans d'un peu plus de 0,3 (soit un développement humain faible) à 0,794 rejoignant presque la catégorie des pays au développement humain élevé $(0,8)$.

4. L'étain et le caoutchouc représentent à eux seuls $85 \%$ des exportations malaysiennes en 1957. Si on leur additionne l'huile de palme, le bois et le pétrole brut on atteint $97 \%$ des exportations de marchandises.

5. Chang, P., 1971. "Headteacher's responsabilities in Nation-building: the Malaysian Case » Malaysian Journal of Education.

6. Cf. Loh P., 1975. Seeds of Separatism : educational policy in Malaya, 1874-1940.

7. Aujourd'hui, son développement humain est dit moyen (IDH =0,794), mais il est très proche du niveau de développement humain élevé (IDH =0,8). Banque Mondiale: Rapport sur le développement dans le monde, 1999-2000.

8. Mingat, Tan, 1992.

9. Le nombre d'illettrés décroît régulièrement de 2,6 millions en 1970 , soit $30 \%$ de la population totale, à 2 millions en 1995 (10 \%). UNESCO, 1999.

10. Jasbir, Hena 1993. «Education and national integration in Malaysia » International Journal of Educational Development, vol. 13 (2).

11. Cf. Solow 1956, 1957.

12. [Romer 1990] désigne l'article de théorie de la croissance endogène portant pour la première fois sur l'innovation et le progrès technique : ROMER P., 1990. «Endogenous Technical Change », Journal of Political Economy, 98, October. pp. 71-102.

13. Cluster: terme d'économie géographique pour désigner un regroupement d'entreprises (le plus souvent appartenant à une même branche de l'industrie) sur un territoire donné. 
INDEX

Index géographique : Malaisie

Mots-clés : développement économique, politique de développement, politique éducative

\section{AUTEUR}

\section{ELSA LAFAYE DE MICHEAUX}

Elsa Lafaye de Micheaux est maître de conférences à l'IUT d'Angers, chercheur associé au MATISSE, UMR-CNRS, Paris 1 [elsaldm@aol.com]. 